\title{
Lesão medular no Centro de Reabilitação e Readaptação Dr. Henrique Santillo (CRER- GO)
}

\author{
Spinal cord injury in Dr. Henrique Santillo Rehabilitation and \\ Readaptation Center (CRER-GO) \\ Lesión medular en el Centro de Rehabilitación y Readaptación \\ Dr. Henrique Santillo (CRER-GO)
}

\author{
Natália Ribeiro de Oliveira Custódio' \\ Maurício Rassi Carneiro' \\ Caroline Campelo Feres' \\ Gabriela Henrique Souza Lima' \\ Maria Raquel Ramos Jubé ${ }^{1}$ \\ Leonardo Eizo Watanabe ${ }^{1}$ \\ Lívia Gabriela Rocha Santos de Oliveira Saliba' \\ Sérgio Daher ${ }^{2}$ \\ Ana Cristina Ferreira Garcia ${ }^{3}$
}

\section{RESUMO}

Objetivo: avaliação epidemiológica de pacientes com diagnóstico de lesão medular internados para reabilitação na enfermaria do Centro de Reabilitação e Readaptação Dr. Henrique Santillo (CRER) em Goiânia, Goiás. Métodos: estudo epidemiológico retrospectivo baseado na coleta de dados dos prontuários médicos dos pacientes internados no período de Março de 2007 a Março de 2009. Resultados: foram avaliados 208 pacientes, com predomínio do sexo masculino $(78,85 \%)$ e idade média de 35,3 anos. O déficit neurológico mais prevalente foi paraplegia $(64,90 \%)$, sendo $54,33 \%$ dos pacientes classificados como ASIA A (lesão completa) de acordo com a classificação da American Spinal Injury Association (ASIA). O tempo médio de lesão até a admissão no CRER foi de 129,7 dias, e o tempo médio de internação

\begin{abstract}
Objective: retrospective epidemiological analysis of spinal cord injury patients admitted to Centro de Reabilitação e Readaptação Dr. Henrique Santillo (CRER) in Goiânia, Goiás. Methods: retrospective epidemiological study based on the analysis of medical records data of patients who were hospitalized in the period from March 2007 to March 2009. Results: 208 patients were analyzed, predominantly male $(78.85 \%)$ with a mean age of 35.3 years. The most neurological deficit was paraplegic $(64.90 \%)$, and $54.33 \%$ of the patients were classified as ASIA $A$ (complete lesion), according to the American Spinal Injury Association (ASIA). The average time from injury to admission was 129.7 days, and the mean time of hospitalization was 44.9 days. The main cause was traumatic spinal cord injury and of these, $44.70 \%$ were from traffic accidents. The main
\end{abstract}

\section{RESUMEN}

Objetivo: evaluación epidemiológica de pacientes con diagnóstico de lesión medular internados para rehabilitación en urgencias del Centro de Rehabilitación y Readaptación Dr. Henrique Santillo (CRER) en Goiânia, Goiás. Métodos: estudio epidemiológico retrospectivo con base en la recogida de datos de las historias médica de los pacientes internados en el periodo de Marzo de 2007 a Marzo de 2009. Resultados: fueron evaluados 208 pacientes, con predominio de sexo masculino $(78.85 \%)$, edad promedio de 35.3 años. El déficit neurológico más relevante fue paraplejia (64.90\%), siendo $54.33 \%$ clasificados como ASIA A (lesión completa) de acuerdo con la clasificación de la American Spinal Injury Association (ASIA). El tiempo promedio de una lesión a la admisión en el CRER fue de 129.7 días y media estancia en el hospital para la reha-

\footnotetext{
Trabalho realizado no Centro de Reabilitação e Readaptação Dr. Henrique Santillo - CRER - Goiânia (GO), Brasil.

'Residentes de Medicina Física e Reabilitação do Centro de Reabilitação e Readaptação Dr. Henrique Santillo - CRER - Goiânia (GO), Brasil.

${ }^{2}$ Médico Ortopedista, Diretor do Centro de Reabilitação e Readaptação Dr. Henrique Santillo - CRER - Goiânia (GO), Brasil.

${ }^{3}$ Médica Fisiatra do Centro de Reabilitação e Readaptação Dr. Henrique Santillo - CRER - Goiânia (GO), Brasil. 
para reabilitação destes pacientes foi de 44,9 dias. A principal etiologia da lesão medular foi traumática, sendo $44,70 \%$ representadas por acidente de trânsito. A complicação mais comum na internação foi infecção do trato urinário. Conclusão: os dados epidemiológicos de pacientes com lesão medular, admitidos no CRER para reabilitação, se mostraram em conformidade com dados já descritos na literatura, demonstrando cada vez mais a importância da divulgação e realização de campanhas de prevenção do trauma raquimedular. Assim como a necessidade do encaminhamento precoce destes pacientes ao centro de reabilitação, uma vez que a grande maioria é jovem, com sequelas neurológicas graves e altamente incapacitantes.

\section{DESCRITORES: Traumatismos}

da medula espinhal;

Traumatismos da medula espinhal/prevenção \& controle; Traumatismos da medula espinhal/epidemiologia; Saúde pública; Acidentes de trânsito/ reabilitação complication was urinarytractinfection. Conclusion: the epidemiological data of spinal cord injury patients admitted to rehabilitation in CRER was in accordance with data already reported in literature, demonstrating the importance of dissemination and implementation campaigns for the prevention of spinal cord trauma. As well as the need for early referral of patients to a rehabilitation center, since most of them are young with severe neurological deficit and highly disabling.

KEYWORDS: Spinal cord injuries; Spinal cord injuries/prevention \& control; Spinal cord injuries/epidemiology; Public health; Accidents, traffic/rehabilitation bilitación de estos pacientes fue de 44.9 días. La principal etiología de la lesión medular fue traumática, siendo $44.70 \%$ representados por accidente de tráfico. La complicación más común en la internación fue infección del tracto urinario. Conclusión: los datos epidemiológicos de pacientes con lesión medular admitidos en el CRER para rehabilitación se mostraron en conformidad con aquellos ya descritos en la literatura, demostrando cada vez mayor importancia de la divulgación y realización de campañas de prevención del trauma raquimedular. También hay necesidad de remitir de forma precoz a estos pacientes al centro de rehabilitación, una vez que la gran mayoría son pacientes jóvenes, con secuelas neurológicas graves y altamente discapacitantes.

DESCRIPTORES: Traumatismos de la médula espinal; Traumatismos de la médula espinal/prevención \& control; Traumatismos de la médula espinal/epidemiología; Salud pública; Accidentes de tráfico/ rehabilitación

\section{INTRODUÇÃO}

A lesão medular (LM), especialmente a de origem traumática, é uma lesão neurológica incapacitante, com grande impacto na sociedade, representando um problema de saúde pública. A incidência desse tipo de lesão é bastante variada nos diferentes países, e ainda não é bem definida no Brasil por não ter uma notificação eficaz dos casos e pela escassez de estudos epidemiológicos significativos ${ }^{1-3}$.

A literatura descreve predomínio no sexo masculino, na faixa etária jovem (entre 15 e 40 anos), causando grande impacto socioeconômico por atingir preferencialmente pessoas em idade produtiva ${ }^{1-4}$.

Acidentes automobilísticos, queda de altura, acidente por mergulho em águas rasas e ferimentos por arma de fogo (FPAF) são as principais etiologias no $\mathrm{Brasil}^{3-4}$, demonstrando que a maioria dos casos de trauma raquimedular (TRM) são traumáticos e passíveis de prevenção. Pode-se citar a realização de campanhas de segurança no trânsito com maior fiscalização e medidas de prevenção à violência urbana ${ }^{5}$.

Atualmente, a reabilitação para os pacientes portadores de LM avançou muito. A expectativa de vida dos lesados medulares é semelhante à de um indivíduo normal, com taxas de morbidades relacionadas à deficiência cada vez mais baixas. Porém, como ainda não há tratamento efetivo para restaurar funções perdidas pela medula comprometida, a reabilitação e readaptação dos pacientes por meio de uma equipe interdisciplinar se tornaram fase obrigatória do tratamento da LM. Isto confirma que o mais importante é realmente conscientizar a população sobre as principais causas de TRM e métodos de prevenção ${ }^{3}$.

O objetivo deste trabalho é traçar o perfil epidemiológico de pacientes com LM, reabilitados no Centro de Reabilitação e Readaptação Dr. Henrique Santillo (CRER), de Março de 2007 a Março de 2009, assim como as principais complicações apresentadas por estes pacientes.

\section{MÉTODOS}

Realizou-se análise retrospectiva de dados dos prontuários médicos dos pacientes com diagnóstico de LM, os quais foram admitidos no serviço de internação do CRER no período de Março de 2007 a Março de 2009 para reabilitação.

\section{RESULTADOS}

Foram avaliados 225 prontuários, do quais 180 eram homens e 45 mulheres. Porém, excluíram-se 17 pacientes 
dentre os quais, oito por dados incompletos e nove devido ainda estarem internados na instituição até o término do presente estudo.

A amostra obtida foi de 208 pacientes, dentre os quais 164 masculinos $(78,85 \%)$ e 44 femininos $(25,15 \%)$, com média de idade de 35,36 anos. Dentre esses, 73 eram tetraplégicos $(35,10 \%)$ e 135 paraplégicos $(64,90 \%)$. A etiologia mais comum da LM foi o acidente no trânsito com 93 pacientes $(44,70 \%)$, sendo $56(26,92 \%)$ vítimas de acidentes por motocicleta e $37(17,78 \%)$ por carro (Gráfico 1$)$. A segunda etiologia mais observada foi a lesão por FPAF, que atingiu 30 indivíduos (14,42\%). Foram admitidos 113 pacientes $(54,33 \%)$ com LM completa, ou seja, ASIA A, representando a maioria da amostra estudada (Gráfico 2). O tempo médio de lesão até a admissão foi de 129,72 dias e de internação na mencionada instituição de 44,91 dias. A complicação mais comum durante a internação destes pacientes foi a infecção do trato urinário (ITU), a qual

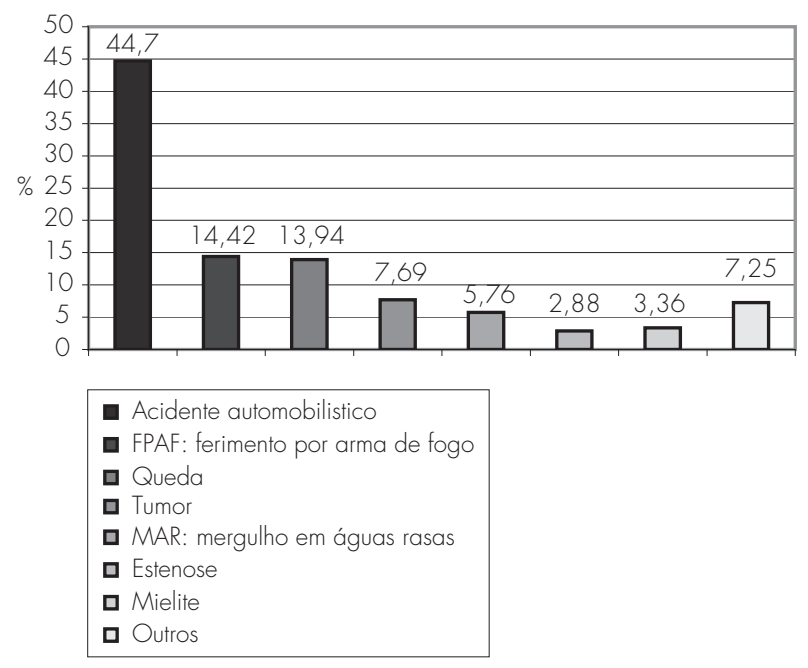

Gráfico 1

Etiologia.

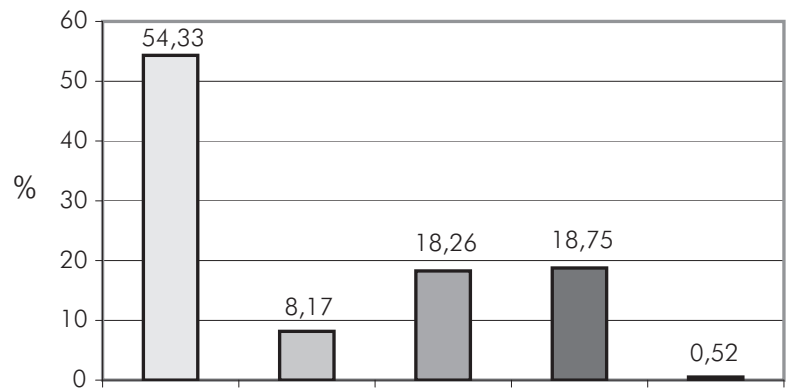

$$
\begin{aligned}
& \square A \\
& \square B \\
& \square C \\
& \square D \\
& E
\end{aligned}
$$

Gráfico 2

Nível neurológico da lesão - ASIA. acometeu 144 (69,23\%) pacientes (Gráfico 3). Um total de 127 pacientes $(61,06 \%)$ usava sonda vesical de demora no momento da admissão e $173(83,17 \%)$ realizavam cateterismo vesical intermitente (CVI) durante a internação. A grande maioria dos pacientes são admitidos no CRER com sonda vesical de demora (o que explica o alto índice de ITU) e, durante a internação, quando indicados, são submetidos a treinamento de CVI pela equipe de Enfermagem.

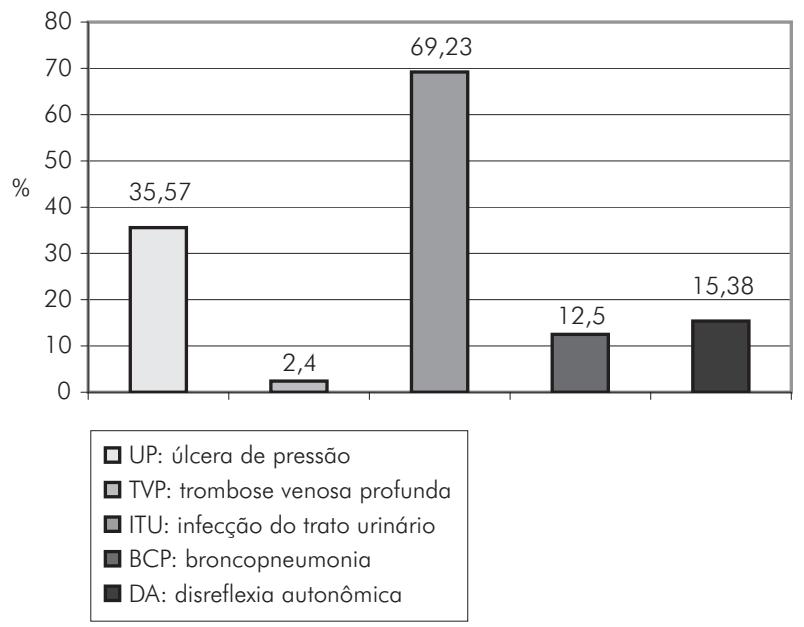

Gráfico 3

Complicações.

\section{DISCUSSÃO}

A LM é uma síndrome incapacitante e um desafio à reabilitação por resultar em alterações motoras, sensitivas e orgânicas em pacientes muito jovens $\mathrm{s}^{6-8}$. A incidência anual de TRM é de 40 casos por milhão na população norte-americana ${ }^{9}$. A região cervical e a transição toracolombar são os seguimentos da coluna mais atingidos, como demonstra o Gráfico 4. Um estudo epidemiológico realizado na cidade de São Paulo apontou como causa predominante de TRM, as quedas ao solo e a média de idade foi de 35 anos $^{4}$. Ape-

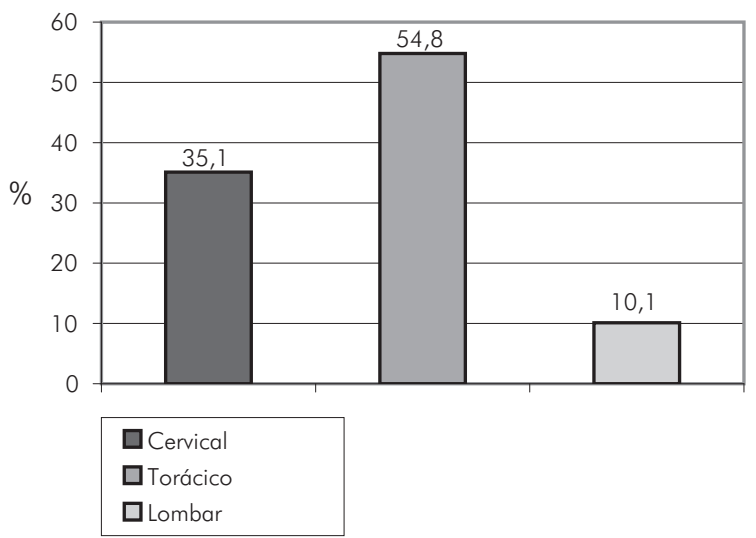

Gráfico 4

Nível ósseo da lesão medular. 
sar das diferenças regionais, este trabalho também mostrou maior prevalência da LM em adultos jovens do sexo masculino, apresentando como causas principais o trauma (acidente automobilístico, seguido de FPAF), sendo a paraplegia e a lesão completa os déficits mais comuns.

O tempo de internação para reabilitação ainda é um dado de difícil comparação na literatura no Brasil, devido ao baixo número de centros de reabilitação que contam com serviço de internação para reabilitação. O tempo de chegada do paciente ao tratamento de reabilitação é bem menor (4,3 meses) do que o citado por Gaspar et al. ${ }^{4}(22,4$ meses), pois os pacientes assim que estáveis clinicamente, são transferidos diretamente dos hospitais gerais para o início mais precoce da reabilitação no CRER. Isto é de extrema importância para a prevenção de complicações secundárias e conscientização da melhor qualidade de vida do paciente incapacitado.

Dentre as complicações, Gaspar et al. citam a úlcera de pressão como a complicação mais frequente, presente em $36 \%$ dos $\operatorname{casos}^{4}$. A prevalência dessas complicações na literatura é bastante variada, de 2,7 a $29,5 \%{ }^{10}$. Esta alta incidência de úlceras de pressão e de outras complicações pode ser a responsável pela elevada média de dias de internação primária encontrada nos trabalhos. Por sua vez, refletindo o despreparo que a grande maioria de nossos hospitais tem em receber o paciente deficiente físico com $\mathrm{LM}^{6}$. Como a presente amostra é composta por pacientes que chegam mais precocemente ao centro de reabilitação, a úlcera de pressão apareceu como segunda complicação mais comum, sendo a ITU a mais observada. Este dado reflete a presença comum da alteração esfincteriana como déficit pós-LM ${ }^{10}$. É acentuada, mais uma vez, a dificuldade dos hospitais em lidar com sequelas decorrentes da LM, deixando os pacientes com sonda vesical de demora por tempo excessivo.

\section{CONCLUSÃO}

O perfil epidemiológico dos pacientes portadores de LM no CRER no período analisado se mostrou em conformidade com os dados já descritos na literatura, expondo cada vez mais a importância da divulgação e realização de campanhas de prevenção do TRM para a população brasileira. Assim como a necessidade de encaminhamento precoce destes pacientes ao centro de reabilitação, uma vez que a grande maioria é jovem com sequelas neurológicas graves e altamente incapacitantes.

\section{REFERÊNCIAS}

1. Barros Filho TEP, Taricco MA, Oliveira RP, Greve JMD, Santos LCR, Napoli MM. Estudo epidemiológico dos traumatismos da coluna vertebral com déficit neurológico internados no Instituto de Ortopedia e Traumatologia do Hospital das Clínicas da Faculdade de Medicina da Universidade de São Paulo. Rev Hosp Clin Fac Med São Paulo. 1990;45(3):123-6.

2. Campos MF, Ribeiro AT, Listik S, Pereira CAB, Sobrinho JA, Rapoport A. Epidemiologia do traumatismo da coluna vertebral. Rev Col Bras Cir. 2008;35(2):88-93.

3. Defino HLA. Trauma raquimedular. Medicina (Ribeirão Preto). 1999;32(4):388-400.

4. Gaspar AP, Ingham SJM, Vianna PCP, Santos FPE, Chamlian TR, Puertas EB. Avaliação epidemiológica dos pacientes com lesão medular atendidos no Lar de São Francisco. Acta Fisiátrica. 2003;10(2):73-7.
5. Gonçalves AMT, Rosa LN, D'Ângelo CT, Savordelli CL, Bonin GL, Squarcino IM, et al. Aspectos epidemiológicos da lesão medular traumática na área de referência do Hospital Estadual Mário Covas. Arq Méd ABC. 2007;32(2):64-6.

6. Souza Junior MF, Neves ACA, Medeiros AAA, Jallageas DN. Características epidemiológicas do trauma raquimedular na Amazônia: Análise prospectiva de 250 casos. J Bras Neurocir. 2003;14(3):97-104.

7. Maia C, Padula MPC. Caracterização dos pacientes lesados medulares que freqüentaram um centro de reabilitação público na cidade de São Paulo. Med Reabil. 2008;27(1):24-8.

8. Siscão MP, Pereira C, Arnal RLC, Foss MHDA, Marino LHC. Trauma raquimedular: caracterização em um Hospital Público. Arq Ciênc Saúde. 2007;14(3):145-7.
9. National Spinal Cord Injury Statistical Center, University of Alabama at Birmingham, 2005. Annual Statistical Report, July, 2005.

10.Taricco MA. Etiologia das lesões medulares. In: Greve JMA, Casalis MEP, Barros TEP, editores. Diagnóstico e tratamento da lesão da medula espinal. São Paulo: Roca; 2001. p. 1-8.

\section{Correspondência}

Ana Cristina Ferreira Garcia

Avenida José Monteiro, 1.655 - Setor

Negrão de Lima

CEP: 74653-230 - Goiânia (GO), Brasil

E-mail: anacgarcia48@yahoo.com.br 